Under consideration for publication in Journal of Colloids and Interface Science

\title{
Hydrodynamics of Bounded Vertical Film with Nonlinear Surface Properties
}

\author{
A. Homayoun Heidari ${ }^{1, *}$, Richard J. Braun ${ }^{2, \S}$, Amir H. Hirsa ${ }^{1}$, Steven A. Snow ${ }^{3}$ and Shailesh Naire ${ }^{4}$ \\ ${ }^{1}$ Department of Mechanical Engineering, Aeronautical Engineering and Mechanics, Rensselaer Polytechnic Institute, Troy, NY \\ $12180 ;{ }^{2}$ Department of Mathematical Science, University of Delaware, Newark, DE 19716; ${ }^{3}$ Interfacial Expertise Center, Dow \\ Corning Incorporation, Midland, MI 48686-0994; and ${ }^{4}$ Mathematical Science Department, Worcester Polytechnic Institute, \\ Worcester, MA 01609
}

\begin{abstract}
The drainage of a thin liquid film with an insoluble monolayer down a vertical wall is studied. Lubrication theory is used to develop a model where the film is pinned at the top with a given thickness and the film drains into a bath at the bottom. A nonlinear equation of state is used for the surface tension and the surface viscosity is a nonlinear function of the surfactant concentration; these are appropriate for some aqueous systems. The three partial differential equations are solved via discretization in space and then solving the resulting differential algebraic system. Results are described for a wide range of parameters, and the conditions under which the free surface is immobilized are discussed.
\end{abstract}

\section{INTRODUCTION}

The drainage of a thin liquid film due to gravity has been studied in a variety of contexts; recent reviews have been given by Oron, Davis and Bankoff (1) and Chang (2). We will use the terminology from Oron et. al (1) to describe a film on a solid substrate as "bounded" and a film bounded only by its own free surfaces as "free". In this work we are interested in the effect of surfactants on bounded film evolution.

The flow down a vertical wall without heating has been studied, among others, by Joo, Davis and Bankoff (3), Joo and Davis (4) and Chang, Demekhin, and Kopelevich (5). Two-dimensional solutions are found to become unstable to three-dimensional waves in the films of infinite extent studied in those papers. The vertical film flow we study in two dimensions here is too short to allow the long wave solutions that they studied; however, it is possible that the solutions studied in this work will be unstable in three dimensions and we will study this in the near future.

\footnotetext{
* Present address: Computational Hydrodynamics Laboratory, Department of Civil \& Environmental Engineering, University of Houston, Houston, TX 77204-4791

$\S$ To whom correspondence should be addressed. Email: braun@math.udel.edu
} 
Modified by numerous applications, including foam stabilization, we have been interested in the drainage of vertically oriented free films with an insoluble surfactant present on the film's surface. In those papers, we studied twodimensional films with a tangentially-immobile surface (6); with constant or linearized surface properties where the average surface tension is important (7) or is negligible (8); and with nonlinear surface properties (9). In those papers, lubrication theory is used to derive evolution equations for the film, and boundary conditions are applied at the top and bottom of the film. The bottom boundary conditions enforce the conditions for a static meniscus at a single point; this is a model for connecting the film to the bath. The models have the advantage of computing the evolution of the entire film while not computing the flow in the bath at the bottom. However, the bottom boundary conditions err on the side of enhancing the surfactant effects there under some conditions. The enhancement of any surfactant effects at the bottom of the film is minimized for the physically common case with nonlinear surface properties in the situations we have studied (9).

Vertical free films draining into a bath have also been studied by Stein and coworkers in an effort to measure and explain the film shapes and the complex flows both in the middle and at the edge of a vertical film in a finite width window (10). They developed quasistatic equations for the free surface shape in their model. Nierstrasz and Frens $(11,12)$ developed a model that focuses on the region that connects the film and the bath, and they do not compute a solution for the whole film. They also have discontinuities in the dependent variables' slopes within the computational domain. They speculate that competition between gravity and the Marangoni effect is responsible for complex flows observed at the film-bath junction, which may be called "peacock feathers" and occur only in vertical films (13).

Bounded films on a vertical wall entering a bath have been studied by a number of workers; for example, in the context of coating flows, see Kheshgi, Kistler and Scriven (14) and the review by Smith (15). In Kheshgi et. al., falling films are studied with different end conditions; these end conditions represent different coating processes and the infinite bath surface that we consider is a special case of what they studied. They did not consider surfactant transport, however. The drag-out problem with surfactant was analyzed using matched asymptotic expansions by Park (16); he studied the case where the film is dragged vertically out of the bath whereas we are interested in the case where the film flows down a stationary wall into the bath. The case where a film flows down the slightly inclined wall into a bath with evaporation was studied by Hosoi and Bush (17). They found that there was an instability transverse to the flow into the bath that was due to evaporation. 
The nonlinear surface properties used in Naire et. al. (9) were based on measurements by Lopez and Hirsa (18) for hemicyanine dye, which forms an insoluble monolayer on water. The surface tension is essentially constant for low surface concentration of surfactant, and then it rapidly decreases about 10\% in magnitude near the concentration where a phase transition occurs. The surface shear viscosity has an exponential dependence on the surface concentration in the same interval. The incorporation of those properties causes more localized variation in the surface velocity and film shape than would otherwise be seen. This localization should be observable in experiment.

Toward that end, we now wish to combine the mathematics of these previous works with the detailed interfacial property measurements reported in Lopez and Hirsa (18). We develop a model that is general enough to handle many equations of state, including those of the type mentioned above. Lubrication theory is used to derive equations that govern the film evolution in two dimensions; the equations will determine the location of the film surface, the surface concentration of the insoluble surfactant, and the surface velocity along the film. The parameters that are representative for a class of surfactants like hemicyanine will be used, and the effect of varying some of the parameters studied.

\section{FORMULATION}

\subsection{Dimensional Problem}

A two-dimensional thin liquid film draining down a rigid vertical wall is modeled (see Figure 1). The Cartesian coordinate system is considered such that $\bar{x}$ is along the film thickness and $\bar{z}$ is along the gravity vector, so $\mathbf{g}=g \mathbf{k}$, where $\mathbf{k}$ is the unit vector along $\bar{z}$ and $g$ is the gravity constant (the bar over variables indicates dimensional). The free surface of the film is defined by $\bar{x}=\bar{h}(\bar{z}, \bar{t})$ and it is assumed a constant at the top of the film, $\bar{h}(0, \bar{t})=h_{0}$. The hydrodynamics of the film is governed by the incompressible Navier-Stokes equations

$$
\begin{gathered}
\bar{\nabla} \cdot \overline{\mathbf{v}}=0, \\
\rho\left(\frac{\partial \overline{\mathbf{v}}}{\partial \bar{t}}+\overline{\mathbf{v}} \cdot \bar{\nabla} \overline{\mathbf{v}}\right)=\mu \bar{\nabla}^{2} \overline{\mathbf{v}}-\bar{\nabla} \bar{p}+\rho g \mathbf{k},
\end{gathered}
$$

where [1] is the continuity equation and [2] is the vector momentum equation. Here $\overline{\mathbf{v}}=(\bar{u}, \bar{w})$ is the velocity vector, $\rho$ and $\mu$ are the density and dynamic shear viscosity of the bulk liquid, respectively, and $\bar{p}$ is the pressure. The boundary conditions applied on the above equations is the no-slip and no-flux condition at the wall defined by 


$$
\bar{u}=\bar{w}=0, \quad \text { at } \bar{x}=0
$$

Also the kinematic boundary condition on the free surface defines the instantaneous location of the surface as

$$
\bar{u}=\frac{\partial \bar{h}}{\partial \bar{t}}+\bar{w} \frac{\partial \bar{h}}{\partial \bar{z}}
$$

Based on the Boussinesq-Scriven surface stress model $(19,20)$ the normal stress boundary condition at the free surface, for a one-dimensional interface, is given by Edwards et al. (21) and Slattery (22)

$$
-\overline{\mathbf{n}} \cdot\|\overline{\mathrm{T}}\| \cdot \overline{\mathbf{n}}=2 \overline{\mathrm{H}} \bar{\sigma}+2 \overline{\mathrm{H}}\left(\kappa^{s}+\mu^{s}\right) \overline{\nabla_{s}} \cdot \overline{\mathbf{v}}
$$

Here, $\overline{\mathbf{n}}$ is the unit outward surface normal vector, $\overline{\mathrm{H}}=\overline{\mathbf{I}}_{s}: \mathbf{b}$ is the mean curvature of the free surface, $\overline{\mathbf{I}}_{s}=\mathbf{I}-\overline{\mathbf{n}} \overline{\mathbf{n}}$ is the spatial idem factor, $\overline{\mathbf{b}}=-\bar{\nabla}_{s} \overline{\mathbf{n}}$ is the surface curvature dyadic, $\bar{\nabla}_{s}$ denotes the surface gradient operator, $\bar{\sigma}$ is the surface tension, $\kappa^{s}$ and $\mu^{s}$ are the surface dilatational and shear viscosity, respectively, and $\|\overline{\mathrm{T}}\|=\overline{\mathrm{T}}_{a}-\overline{\mathrm{T}}$ denotes the jump in the stress tensor across the free surface, where $\overline{\mathrm{T}}_{a}$ is the stress tensor in the gas (air) phase and is assumed to be zero in this model. $\bar{T}$ is defined by

$$
\overline{\mathrm{T}}=-\mathbf{I} \bar{p}+\mu\left(\bar{\nabla} \overline{\mathbf{v}}+\bar{\nabla} \overline{\mathbf{v}}^{t}\right)
$$

The tangential stress boundary condition for a one-dimensional interface with variable surface viscosities, based on the above model, is defined as follows $(21,22)$

$$
-\overline{\mathbf{t}} \cdot\|\overline{\mathrm{T}}\| \cdot \overline{\mathbf{n}}=\overline{\mathbf{t}} \cdot \bar{\nabla}_{s} \bar{\sigma}+\left(\kappa^{s}+\mu^{s}\right) \overline{\mathbf{t}} \cdot \bar{\nabla}_{s} \bar{\nabla}_{s} \cdot \overline{\mathbf{v}}+\overline{\mathbf{t}} \cdot \bar{\nabla}_{s}\left(\kappa^{s}+\mu^{s}\right) \bar{\nabla}_{s} \cdot \overline{\mathbf{v}}
$$

where $\overline{\mathbf{t}}$ is the unit tangent vector along the free surface. The last term in [7] accounts for the possibly strong variability of the surface viscosities with surfactant surface concentration. The surface differentiation operators follow Scriven (20), Stone (23) and Wong et al. (24).

The concentration of the insoluble surfactant on the free surface is governed by the following advectiondiffusion equation $(20,23)$

$$
\frac{\partial \bar{\Gamma}}{\partial \bar{t}}+\bar{\nabla}_{s} \cdot(\bar{\Gamma} \overline{\mathbf{v}})=\mathrm{D}_{s} \bar{\nabla}_{s}^{2} \bar{\Gamma}
$$

where $\bar{\Gamma}=\bar{\Gamma}(\bar{z}, \bar{t})$ is the surface concentration of the insoluble monolayer of surfactant; and $\mathrm{D}_{s}$ is the surface diffusivity. 


\subsection{Surface Properties}

The surface tension, for a fluid containing a dilute insoluble surfactant, is no longer a constant value. There are a number of mathematical models presented for the surface tension in the presence of different surfactants. Lopez and Hirsa (18) introduced the following expression for hemicyanine, an insoluble monolayer used in several experiments,

$$
\begin{gathered}
\bar{\sigma}(\Gamma)=\sigma_{m}+\beta F(\alpha, \Gamma), \\
F(\alpha, \Gamma)=\tanh [\alpha(\Gamma-1)], \quad(\alpha<0),
\end{gathered}
$$

where $\sigma_{m}$ is a characteristic surface tension, $\Gamma=\bar{\Gamma} / \Gamma_{m}$ is the nondimensional surfactant concentration, and $\Gamma_{m}$ is the reference surfactant concentration, and $\beta$ and $\alpha$ are a dimensional and a nondimensional fitting parameter, respectively. For the same surfactant, an analytical fit for the surface shear viscosity is also given by Lopez and Hirsa (18)

$$
\mu^{s}=\mu_{m}^{s}(-1+\exp (\gamma \Gamma))
$$

where $\mu_{m}^{s}$ is the reference surface shear viscosity, and $\gamma$ is a nondimensional parameter. The surface dilatational viscosity is not yet been measure consistently with any two different experimental techniques (21) and it is usually modeled as a multiple of surface shear viscosity. A wide range for the ratio of $\theta=\kappa^{s} / \mu^{s}$ have been suggested in various works, e.g. 2:1 (25) to 2000:1 (26). However, we will consider this ratio as a variable and will discuss its effect in the parameter study of the results. Taking this ratio into account, equation [11] may be written as follows for the total surface viscosity

$$
\left(\kappa^{s}+\mu^{s}\right)=(\theta+1) \mu_{m}^{s}(-1+\exp (\gamma \Gamma)) .
$$

\subsection{Nondimensional Problem}

The length scales for the nondimensionalization are based on the tangentially immobile unbounded film $(27,28)$. Three scales are introduced

$$
d=\sqrt{\frac{\mu W}{\rho g}}, D=\sqrt{\frac{\bar{\sigma}}{\rho g}}, \text { and } \ell=\mathrm{d}^{\frac{1}{3}} D^{\frac{2}{3}} .
$$


Which are, respectively, the average initial film thickness, equilibrium meniscus radius, and the intermediate scale, and are also depicted in the schematic of the model (FIGURE 1). $W$ is the vertical velocity scale which is defined from [13], having the value $d$. Separation of scales occurs when the ratio of length scales is very small, i.e. $\delta^{2}=d / \ell \square 1$

Using the above length and velocity scales, the following nondimensional variables are introduced into the problem

$$
x=\frac{\bar{x}}{d}, z=\frac{\bar{z}}{\ell}, t=\frac{W}{\ell} \bar{t}, u=\frac{\bar{u}}{\delta^{2} W}, w=\frac{\bar{w}}{W}, \text { and } p=\frac{\delta^{4} \ell}{\mu W} \bar{p} .
$$

Based on the above nondimensionalization, the motion equations inside the film are furnished as follows

$$
\begin{gathered}
u_{x}+w_{z}=0, \\
\delta^{8} \mathrm{R}\left(u_{t}+u u_{x}+w u_{z}\right)=\delta^{4}\left(u_{x x}+\delta^{4} u_{z z}\right)-p_{x}, \\
\delta^{4} \mathrm{R}\left(w_{t}+u w_{x}+w w_{z}\right)=w_{x x}+\delta^{4} w_{z z}-p_{z}+1,
\end{gathered}
$$

these the continuity equation and the momentum equation for $x$ and $z$ directions, respectively, and $\mathrm{R}$ is the Reynolds number,

$$
\mathrm{R}=\frac{\rho W \ell}{\mu} .
$$

The nondimensional surfactant transport equation [8] will be given by

$$
\Gamma_{t}+N^{2}\left[\delta^{2} h_{z} \mathrm{~A}(\Gamma u)+\mathrm{A}(\Gamma w)\right]=\frac{1}{\mathrm{P}}\left[\left(\delta^{2} N^{2} h_{z} \mathrm{~A}\right)^{2}+\left(N^{2} \mathrm{~A}\right)^{2}\right] \Gamma,
$$

where the Peclet number is defined by

$$
\mathrm{P}=\frac{W \ell}{\mathrm{D}_{s}} .
$$

Also, the following definitions are used in [19]

$$
N=\frac{1}{\sqrt{1+\delta^{2} h_{z}^{2}}}, \mathrm{~A} \equiv h_{z} \frac{\partial}{\partial x}+\frac{\partial}{\partial z} .
$$

The nondimensional kinematic boundary condition becomes

$$
h_{t}-u+h_{z} w=0 .
$$


For the sake of generality, the surface property functions will be rewritten based on [12] as follows in the interfacial stress components nondimensional formulation

$$
\left(\kappa^{s}+\mu^{s}\right)=(1+\theta) \mu_{m}^{s} \Omega(\Gamma)
$$

where $\Omega(\Gamma)$ is a nondimensional function of surfactant concentration. The nondimensional normal component of the interfacial stress, is given by

$$
\begin{gathered}
-p+2 N^{2} \delta^{4}\left[u_{x}-h_{z}\left(\delta^{4} u_{z}+w_{x}\right)+\delta^{4} h_{z}{ }^{2} w_{z}\right]= \\
N^{3} h_{z z}\left[\frac{\delta^{6}}{\mathrm{C}}+\delta^{4} \frac{\mathrm{M}}{\alpha} F+\delta^{4} N^{2} \mathrm{~S} \Omega(\Gamma)\left(\delta^{2} h_{z} u_{z}+w_{z}\right)\right],
\end{gathered}
$$

where the following nondimensional groups are used

$$
\mathrm{M}=\frac{-\left(\frac{\partial \sigma}{\partial \Gamma}\right)_{\Gamma_{m}} \cdot \Gamma_{m}}{\mu W} \delta^{2}, \mathrm{C}=\frac{\mu W}{\sigma_{m}}, \text { and } \mathrm{S}=\frac{(1+\theta) \mu_{m}^{s}}{\ell W} \delta^{2}
$$

where these numbers are Marangoni, capillary, and modified Boussinesq, respectively. It should be noted that for our scaling, $C=\delta^{6}$. The nondimensional tangential component of interfacial stress can be written in nondimensional form as

$$
\begin{gathered}
N^{2}\left[2 \delta^{4} h_{z}\left(u_{x}-w_{z}\right)+\left(1-\delta^{4} h_{z}^{2}\right)\left(\delta^{4} h_{z}+w_{x}\right)\right]= \\
-N \mathrm{M} \frac{\partial \sigma}{\partial z}+\mathrm{S} \Omega N^{3}\left(\delta^{4} h_{z z} u_{z}+\delta^{4} h_{z} u_{z z}+w_{z z}\right)+\mathrm{S} N^{3} \Omega_{\Gamma} \Gamma_{z}\left(\delta^{4} h_{z} u_{z}+w_{z}\right)
\end{gathered}
$$

In our model, using [9] for the variations of surface tension with surfactant concentration, the Marangoni number would be defined as

$$
\mathrm{M}=\frac{-\beta \alpha}{\mu W} \delta^{2} .
$$

\subsection{Lubrication Theory}

The solution is expanded in the form of a regular perturbation expansion using $\delta^{4}$ as the perturbation coefficient as follows

$$
(\mathbf{v}, h, p, \Gamma)=(\mathbf{v}, h, p, \Gamma)^{(0)}+\delta^{4}(\mathbf{v}, h, p, \Gamma)^{(1)}+\cdots
$$


The above expansion is then substituted into the scaled equations. After dropping the superscripts, the leading order of equations may be written as

$$
\begin{gathered}
u_{x}+w_{z}=0, \\
0=-p_{x}, \\
0=w_{x x}-p_{z}+1 .
\end{gathered}
$$

The boundary condition at the wall, $x=0$, is

$$
u=w=0,
$$

and the boundary conditions on the free surface of the film, $x=h(z, t)$, can be written as

$$
\begin{gathered}
h_{t}-u+h_{z} w=0 \\
\mathrm{~S} \Omega w_{z z}+\mathrm{S} \Omega_{\Gamma} \Gamma_{z} w_{z}-\frac{1}{N} w_{x}-\frac{1}{N^{2}} \mathrm{M} \sigma_{\Gamma} \Gamma_{z}=0 \\
-p=\eta
\end{gathered}
$$

where these equations are the leading order of kinematic, tangential stress, and normal stress boundary conditions, respectively. Here, $\eta$ is the film surface curvature, which is defined by

$$
\eta=N^{3} h_{z z}=\frac{h_{z z}}{\left(1+\delta^{4} h_{z}^{2}\right)^{\frac{3}{2}}} .
$$

Using [31]-[33], the vertical component of velocity vector may be written as

$$
w(x, z, t)=-\left(\eta_{z}+1\right) \frac{x^{2}}{2}+\mathrm{A}(z, t) x
$$

Here $\mathrm{A}(z, t)$ is an unknown function so far. Now defining the vertical surface velocity as

$$
\varpi(z, t)=w(h(z, t), z, t) .
$$

It may now be expressed by the following equation based on [37]

$$
\varpi(z, t)=-\left(\eta_{z}+1\right) \frac{h^{2}}{2}+\mathrm{A}(z, t) h .
$$

The governing equation for the surface velocity will be derived by substituting the above expression into [34]

$$
\mathrm{S}\left(V \varpi_{z}\right)_{z}+\frac{1}{N}\left\{\frac{1}{2}\left(\eta_{z}+1\right) h-\frac{\varpi}{h}\right\}-\frac{1}{N^{2}} \mathrm{M} \sigma_{\Gamma} \Gamma_{z}=0 .
$$


Using the definition for surface velocity, the kinematic boundary condition [33] can be written as follows

$$
h_{t}+\frac{\partial}{\partial z}\left[\frac{1}{2} \varpi h+\frac{1}{12}\left(\eta_{z}+1\right) h^{3}\right]=0 .
$$

These two equations are coupled with the surfactant transportation equation [19], which has the following form in the leading order

$$
\Gamma_{t}+N^{2} \frac{\partial}{\partial z}\left[\Gamma \varpi-\frac{1}{\mathrm{P}} N^{2} \Gamma_{z}\right]=0
$$

Equations [40]-[42], are the three coupled differential equations which should be solved for this mathematical model along with the boundary conditions defined in the subsequent section.

\subsection{Boundary Conditions}

At the top of the film, i.e. $z=0$, we assume that the film is fixed to a film holder, as used in the experiments. Also we assume that the no flux condition holds for the top of the film. In the experiments, this is accomplished by using a film holder made of inert material such as Teflon. These conditions may be summarized into

$$
h(0, t)=0, h_{z}(0, t)=0, \Gamma_{z}(0, t)=0 \text {, and } \varpi(0, t)=0 .
$$

At the bottom of the film, i.e. $z=L$, the film should be matched into the static meniscus which is governed by the Young-Laplace equation,

$$
\eta_{z}=-1
$$

The solution for a two-dimensional case is parameterized by $v$,

$$
\frac{v}{\delta^{2}}=\eta^{2}+\frac{2 h_{z}}{\sqrt{1+\delta^{4} h_{z}^{2}}},
$$

which is a constant in the first integral of [44]. The value of this parameter for an infinite bath, which holds for our case, is $v=2$. However, having a fixed initial condition for $h_{z}$ at the bottom of the film, we can define $h_{z z}$ using [45] by the equation

$$
h_{z z}=\left[2\left(1+\delta^{4} h_{z}^{2}\right)^{3}\left(\frac{1}{\delta^{2}}-\frac{h_{z}}{\sqrt{1+\delta^{4} h_{z}^{2}}}\right)\right]^{\frac{1}{2}} .
$$


Imposing $h_{z}$ and $h_{z z}$ at the end of the domain is an asymptotic "patch" because the solutions only agree at a single point. In standard matched asymptotics, there is a finite region of overlap between the two solutions. The patching will conserve the amount of surfactant over the film region and it conserves surfactant over the entire fluid surface (union of film and bath surfaces) as may be verified by a simple integration along the fluid surface. However, the surfactant concentration may jump across the patch point from the film side to the meniscus side. We choose to use this approach because this choice will accentuate any effect of surfactant build up at the bottom of the film as it is washed down during drainage.

The other boundary conditions at the film end are no flux for both bulk phase and the surfactant,

$$
\varpi(L, t)=0, \Gamma_{z}(L, t)=0,
$$

where $L$ is the length of the film. This furnishes all the necessary boundary conditions that we need at the ends of the film.

\subsection{Initial Conditions}

Linear initial conditions are assumed for the film shape and surfactant distribution. The following form is considered for the film surface

$$
h(0, z)=1+s_{0} z, \quad 0 \leq z \leq L,
$$

where $s_{0}$ is the initial slope. The initial surfactant concentration is chosen to be of the form

$$
\Gamma(0, z)=\hat{\Gamma}+\gamma_{0}\left(z-\frac{L}{2}\right)
$$

where $\hat{\Gamma}$ is the average surfactant concentration and $\gamma_{0}$ is the initial surfactant gradient, i.e. $\gamma_{0}=\left(\Gamma_{\text {top }}-\Gamma_{\text {bottom }}\right) / L$.

\section{NUMERICAL SCHEME}

In order to numerically solve the system of nonlinear PDEs of [40]-[42], the spatial variable is discretized using a second-order accurate finite difference scheme and the temporal variable is left continuous. The resulting system may be written in the form of a system of differential-algebraic equations (DAEs) which is then solved by the package DASSL (29).

In order to derive the DAE system, the following discretization in the spatial direction is employed. The kinematic boundary condition [41] is first written in terms of flux as follows

$$
h_{t}=-\Phi_{z},
$$


where the flux is defined by

$$
\Phi \equiv \frac{1}{2} \varpi h+\frac{1}{12}\left(\eta_{z}+1\right) h^{3}
$$

The conservation of flux over the film region is assured by using the above form for the film evolution equation. Equation [51] is coupled with the surface velocity equation [40] and the surfactant transport equation [42] which the later is again written in terms of surfactant flux as follows

$$
\Gamma_{t}+N^{2} \Psi_{z}=0
$$

where the surfactant flux is defined by

$$
\Psi \equiv \Gamma \varpi-\frac{1}{\mathrm{P}} N^{2} \Gamma_{z}
$$

Three dependent variables which are to be evaluated, namely $h, \varpi$, and $\Gamma$, are then discretized as $h_{i}=h\left(z_{i}, t\right)$, $\varpi_{i}=\varpi\left(z_{i}, t\right)$, and $\Gamma_{i}=\Gamma\left(z_{i}, t\right)$ based on grid points $z_{i}=i \Delta z, i=0, \ldots, M$, where $M$ is the number of grid points.

Up to third-order derivative of film thickness function is needed to calculate the curvature derivative term in [51] , leading to the following finite difference formulation

$$
h_{z z z} \approx \frac{h_{i+2}-2 h_{i+1}+2 h_{i-1}-h_{i-2}}{2(\Delta z)^{3}},
$$

which in turn produces the need for fictitious points $h_{-1}$ and $h_{M+1}$. Based on a perturbation expansion of the boundary condition [44] at the end of the film using $\delta^{4}$ as the perturbation coefficient, the following explicit formulation for $h_{-1}$ may be derived

$$
h_{-1}=h_{-1}^{(0)}+\delta^{4} h_{-1}^{(4)}+O\left(\delta^{8}\right)
$$

where

$$
\begin{gathered}
h_{-1}{ }^{(0)}=\frac{1}{3}\left[2(\Delta z)^{2}+10 h_{0}-12 h_{1}+6 h_{2}-h_{3}\right], \\
h_{-1}{ }^{(4)}=\frac{\Delta z}{4}\left(h_{1}-h_{-1}{ }^{(0)}\right)-\frac{1}{(\Delta z)^{2}}\left(h_{1}-h_{-1}{ }^{(0)}\right)\left(h_{1}+h_{-1}{ }^{(0)}-2 h_{0}\right)^{2} .
\end{gathered}
$$

Also $h_{M+1}$ and $h_{M+2}$ are derived based on the boundary conditions for $h_{z z}$ and $h_{z}$ at the bottom of the computational domain resulting in 


$$
\begin{aligned}
& h_{M+1}=s_{1}(\Delta z)^{2}+2 h_{M}-h_{M-1}, \\
& h_{M+2}=-2 s_{0} \Delta z+4 h_{M+1}-3 h_{M},
\end{aligned}
$$

where $s_{0}$ and $s_{1}$ are the first and second-order derivatives at the bottom of the domain, respectively. Also, fictitious points are needed for the surfactant concentration where they are derived based on the top [43] and bottom [47] boundary conditions. At the bottom of the domain, one-sided difference approximations are used in order obtain flux derivatives as follows

$$
\Phi_{z} \approx \frac{3 \Phi_{M}-4 \Phi_{M-1}+\Phi_{M-2}}{2 \Delta z}
$$

and the same procedure is applied to the surfactant flux, $\Psi$. Having all the dependent variables discretized, the system may now be fed into the DASSL package as the following implicit form

$$
\mathrm{G}(t, y, \dot{y})=0
$$

where a dot denotes a temporal derivative, and $y$ may be either a differential or an algebraic variable. Using a backward difference scheme, the system is discretized in temporal direction is then solved by a modified version of Newton's method.

\section{RESULTS}

In the results to follow, a parametric study is conducted on the hydrodynamic behavior of the thin film modeled in the earlier sections.

The effect of the value of surfactant average concentration is first considered. In this study, a low surface viscous effect and a relatively high Marangoni effect is considered so that the effect of Marangoni-driven flow would be dominant. Next, the effect of initial surfactant concentration gradient is discussed based on a fixed average concentration and other parameters. The behavior of the film is subsequently analyzed in a low-viscous, low-Marangoni effect regime. Then the effect of the surface concentration, both initial distribution and the average concentration is studied in a high surface viscosity situation. A case of high-viscosity and low Marangoni effect is discussed next and finally the similarity behavior of the thin film is studied based on a situation of high Marangoni effect and various levels of surface viscosity effect. 
For all the following results the physical properties of the fluid/surfactant system is considered as follows: $\mu=0.01002 \mathrm{~g} / \mathrm{cm} \cdot \mathrm{s}, \quad \rho=1 \mathrm{~g} / \mathrm{cm}^{3}, \quad d=10 \mu \mathrm{m}, \quad \ell=0.28 \mathrm{~mm}, \quad \beta=6.3$ dyne $/ \mathrm{cm}, \quad \alpha=-6.2, \quad \mathrm{D}_{\mathrm{s}}=10^{-6} \mathrm{~cm}^{2} / \mathrm{s}$, $\delta=0.189$

Based on the above parameters, a Marangoni number of $M=1500$ and a Peclet number of $P=2700$ are used in the following results. Two different values of the ratio of dilatational surface viscosity $\kappa^{s}$ to surface shear viscosity $\mu^{s}$ are used in our computations in order to cover both low and high viscosity. Hence, based on $\theta=10$ and $\theta=1000$, two different values of $S=0.16$ and $S=15$ are used in the computations. Also for limiting cases of surface viscous effects and/or Marangoni effects, other artificial values of $S$ and $M$ are used as described in the following sections.

\subsection{Effect of Average Concentration}

The effect of the average concentration is studied based on the three values of surface concentration $\hat{\Gamma}=0.50,0.65,0.80$, which are considered to be relatively low, medium, and high, respectively (see FIGURE 2 ). In all three cases, other parameters are considered to be fixed at the following values: $S=0.16, M=1500, P=2700$. Therefore, the flow regime may be described as low surface viscosity and high Marangoni effect. Also no initial surfactant concentration gradient is assigned for these cases.

FIGURE 3 illustrates the film behavior having a relatively low average surface concentration of surfactant. At very early times $(t=4)$, upper half of the film has a positive surface velocity distribution therefore the surfactant is being swept down in that region. On the other hand, the lower half has a negative surface velocity distribution, which is negligible compared to that of the upper half. According to FIGURE 2, the dependence of the surface tension on the surfactant concentration in the neighborhood of $\hat{\Gamma}=0.5$, is very weak. Therefore, only around the highgradient area of newly developed surface concentration distribution at $t=4$, a surfactant-driven flow begins to counteract gravity, dragging up the surfactant at later times. However, at the very top of the film, concentration continues to decrease and so does the film thickness due to the downward velocity. A healing effect shapes in the film free surface, moving up with time, around the point where the concentration gradient is high. This upward-traveling wave, is actually dividing the film surface into a rigid, lower part of the film, and a mobile film in the upper part of the film. 
In the next case, illustrated in FIGURE 4, the surface tension is more sensitive to the changes in surfactant concentration distribution due to a higher value of average concentration, $\hat{\Gamma}=0.65$. The surface tension function begins to curve downward in the neighborhood of this value of concentration (see FIGURE 2). Due to this effect, stronger upward flow occurs at the lower $3 / 4$ of the film tending toward redistributing the surfactant along the film height. Also the film thinning is more uniform and slower at the top comparing the previous case due to the negative surface velocities. A very weak wave is observed traveling up the film and gradually disappearing due to smoothed concentration distribution by about $t=32$. Consequently, most of the film surface may be described as rigid in this case.

At the higher average concentration in the third case, $\hat{\Gamma}=0.80$, shown in FIGURE 5 , the surface velocity is completely negative (upward) from early time. No healing is observed on the film surface and the surfactant concentration is symmetrically redistributed after a slight gravitational wash-down effect at $t=4$ in the upper half of the film. The surface tension is highly sensitive to the concentration and this is the reason for the dominance of the Marangoni effect and upward surface flow in this case. Due to the strong Marangoni effect, the film is acting more rigid in this case.

\subsection{The Effect of Initial Concentration Gradient}

By fixing the value of average concentration at $\hat{\Gamma}=0.50$, the effect of initial concentration gradient is discussed in this section. As the first case, the behavior of the film for a relatively low concentration gradient is illustrated inFIGURE 6, using $\gamma_{0}=0.40 / L$. By comparing with the case of FIGURE 3, it is clear that there is no significant difference in the hydrodynamic behavior of the film except that the negative surface velocity is slightly larger in the lower half of the film for the case with an initial gradient in $\Gamma$. This is due to the stronger initial Marangoni effect which pushes the stagnation point on the free surface upward from around $z \square 20$ to $z \square 13$. Consequently, the same wave observed in FIGURE 3 can be identified in this case, starting slightly closer to the top of the film at the first illustrated time step and then continuing to travel upward keeping the same position as the previous case. This is due to the balanced Marangoni effect after the redistribution which starts at about $t=16$. Film may again be described as rigid in the lower part and mobile in the upper part, separated by the wave front. 


\subsection{The Effect of Surface Viscosity}

To study the effect of surface viscosity on the hydrodynamic behavior of the film, the modified Boussinesq number of the previous case illustrated in FIGURE 6, is increased to $S=15$. The Boussinesq number of this case, illustrated in Figure 7, represents an increased value surface viscosity ratio $\theta$ (see equation [12]) from 10 to 1000, resulting in much larger surface dilatational viscosity $\kappa^{s}$ and consequently much stronger surface viscous effects.

The sensitivity of surface tension to the surfactant concentration is relatively low in this case as the curve in FIGURE 2 shows a nearly flat behavior around $\Gamma=0.5$. The significant surface viscous effect has decreased the surface velocity gradient especially around the surface stagnation point, which in turn has made the traveling wave on the surface disappear at the early times. However, as the total Marangoni effect increases at later times the wave starts to form again which can be clearly identified in $t=32$. Still in this case, the Marangoni and viscous effects are weak enough at the very top of the film allowing the surface to get swept clean of the surfactant. The magnitude of the surface velocity peak is approximately $30 \%$ lower than the low surface-viscosity case, making the film surface relatively more sluggish.

\subsection{Limiting Cases for low Marangoni Effect}

Two limiting cases are studied in this section which correspond to a weak Marangoni effect, having either a relatively low or a high Boussinesq number. The first case corresponds to a film with $\mathrm{S}=10$ and $\mathrm{M}=50$, which is illustrated in Figure 8. The average surfactant concentration is set to the flat region of the equation of state and also a relatively mild initial gradient is considered. As it is expected for these parameters, the film is acting completely mobile for the early time. Due to the very weak Marangoni effect, most of the surfactant is swept down and has built up near the meniscus as it is shown in the curves for $t=4$. The surface velocity is also completely positive with the only mechanism acting in this stage being the gravitationally-driven flow. The viscous effects are also small at the top of the film which along with the weak Marangoni effect, causes a rapid thinning in the top of the film. However, the redistributed surfactant concentration enhances the total effective Marangoni and viscous effect and causes the flow to reverse as is illustrated in $t=16$. A wave front forms and starts traveling upward right in the position of the stagnation point which is the equilibrium of the gravitationally- and viscous-driven flow. However, due to low overall Marangoni effect, the traveling wave does not get close to the top of the film for the times we computed and the 
mobile portion of the film is much larger compared to the case with the same strength of viscous effect and higher Marangoni number which is illustrated in Figure 7.

Next, a case with a very strong viscous effect and the same weak Marangoni effect is shown in Figure 9. Due to the highly viscous free surface, the sweeping effect of the surface concentration in the previous case is not observed here. The concentration is gradually reduced in the upper half of the film and starts to buildup in the lower half until $t=128$. At this time, the Marangoni effect starts to turn on due to the adequate concentration gradient which is provided by the downward gravitationally-driven flow. The surface velocity is positive along the film height and no stagnation point is observed. At the last curve shown in the graph which corresponds to $t=512$, a very slight flow reversal starts to occur and the surfactant is driven upward in a small region just over the middle point of the film.

\subsection{Power-Law Behavior of Drainage}

In an attempt to study the similarity behavior of the film drainage, the time-history of the film thickness for three locations along the film height is illustrated in FIGURE 10. Graphs are illustrated for points near the top $(z=L / 4)$, middle $(z=L / 2)$, and bottom $(z=3 L / 4)$ of the film and there are three cases of the surface viscosity strength for each location.

The curves are on a logarithmic scale hence a straight line shows power-law behavior of the film drainage process. The bottom and middle of the film both taken on linear forms with the slope $-\frac{1}{2}$ for; they are essentially rigid for $t \geq 10$. This behavior is consistent with the dominance of the Marangoni effect causing the film to be rigid at these locations. However, the top of film behaves differently and shows more sensitivity to the strength of surface viscous effect. For very high surface viscosity $(S=1500)$, the film acts essentially immobile from very early times, i.e. around. Before this time, mobility is observed on the film surface. For a lower surface viscosity $(S=15)$, the film surface starts by being more mobile up to about $t=20$, and then entering a transition time $20 \leq t \leq 60$.

From approximately $t=60$, the surface becomes immobile and follows a straight line with slope $-\frac{1}{2}$. Therefore, it may be concluded that in this case, the film goes through two transitions. Decreasing the surface viscous effect further by setting $S=0.15$, shows a time interval $4 \leq t \leq 15$ with slope $>-\frac{1}{2}$ which indicates a mobile surface. There follows a transition interval, and for $t \geq 50$ the top part of the film is rigid. 


\section{SUMMARY AND CONCLUSION}

A mathematical model is developed for the hydrodynamic behavior of a thin-liquid film draining over a vertical rigid wall in the regime of both gravitationally- and surfactant-driven flow based on the Boussinesq-Scriven model. Both surface tension and surface viscosities are nonlinearly dependent on the surfactant concentration on the free surface in this model: this follows from a property correlation for a physical insoluble monolayer on water.

Numerical experiments are performed on governing equations to cover the different regimes of the film freesurface and drainage behavior. It is found that for low average surfactant concentration, the top part of the film can be almost swept clean of surfactant. In the same regime, a traveling wave on the film's free surface is observed which acts as a boundary between mobile and rigid behavior of the film. This traveling wave was observed both in low and high surface viscosity cases, but with different phase speed and coverage area. As in the free film case (9), this natural flow can essentially clean the interface when nonlinear properties are present in the surface. Similar behavior has been seen in driven flows as observed for a drop in an extensional flow $(30,31)$ and for flow in a rotating channel viscometer $(32,33)$. In the latter case, the surface becomes immobile in the radial direction but not in the azimuthal direction. In our two-dimensional film, the surface becomes essentially immobile when the surfactant concentration becomes high enough. While the surface of a drop in an extensional flow without nonlinear surface properties can be essentially swept clean (34), in our naturally-driven flows the nonlinear properties appear to be a requirement for this to happen.

It is shown that a surfactant-driven upward flow is caused by having either a high initial gradient of concentration distribution, or a localized gradient after the early-time redistribution due to gravitationally-driven flow.

The surfactant concentration gradient, for an initially linear profile along the film surface, is found to have virtually no effect on the late time film drainage behavior when the average concentration is low enough. The behavior of the film was also studied for a weak Marangoni effect; it was found that even for a weak Marangoni effect the film may act partially rigid and starts to show a healing effect for low surface viscosity. However, by increasing the surface viscosity, the film becomes essentially immobile except for a very localized flow reversal in the middle region of the film at very late times.

Finally, it was also observed that the drainage process shows a power-law behavior in different time periods based on the strength of surface viscous effects. When the Marangoni effect is strong and fixed. However, it was 
generally concluded that all of the film surface thins with power-law behavior at very late times regardless of surface viscous effects.

An experiment is currently being run for comparison with this theory (35). The results will be published in a forthcoming paper.

\section{ACKNOWLEDGMENTS}

R.J. Braun gratefully acknowledges support from Dow Corning Corporation, and the National Science Foundation through grant DMS96-31287. A.H. Heidari is grateful for support from the Department of Mathematical Science at University of Delaware during his extended visit.

\section{REFERENCES}

1. Oron, A., Davis, S. H., and Bankoff, S. G., Reviews of Modern Physics. 69, 931 (1997).

2. Chang, H. -C., Annu. Rev. Fluid Mech. 26, 103 (1994).

3. Joo, S. W., Davis, S. H., and Bankoff, S.G., J. Fluid Mech. 230, 117 (1991).

4. Joo, S. W., Davis, S. H., Chemical Eng. Comm. 118, 111 (1991).

5. Chang, H. -C., Demekhin, E. A., and Kopelevich, D. I., J. Fluid Mech. 250, 443 (1993).

6. Braun, R. J., Snow, S. A., and Pernisz, U. C., J. Colloid Interface Sci. 219, 225 (1999).

7. Naire, S., Braun, R. J., and Snow, S. A., J. Colloid Interface Sci. 230, 91 (2000).

8. Naire, S., Braun, R. J., and Snow, S. A., SIAM J. Appl. Math. 61, 889 (2000).

9. Naire, S., Braun, R. J., and Snow, S. A., Phys. Fluids, to appear. (2001)

10. Stein, H. N., Adv. in Colloid and Interface Sci, 34, 175 (1991).

11. Nierstrasz, V. A., and Frens, G., J. Colloid Interface Sci, 207, 209 (1998).

12. Nierstrasz, V.A., and Frens, G., J. Colloid Interface Sci, 215, 28 (1999).

13. Baets, P.J., and Stein, H.N., Langmui, 8, 3099 (1992).

14. Kheshgi, H. S., Kistler, S. F., and Scriven, L.E., Chem. Eng. Science 47, 683 (1992).

15. Smith, M. K., "Asymptotic methods for the mathematical analysis of coating flows". in "Liquid Film Coating", edited by Kistler, S.F. and Schweizer, P.M. London: Chapman and Hall., 1997.

16. Park, C. W., J. Colloid Interface Sci. 146, 382 (1991).

17. Hosoi, A. E. and Bush, J. W. M., J. Fluid Mech. (2000), in press.

18. Lopez, J. M., and Hirsa, A., J. Colloid Interface Sci. 229, 575 (2000).

19. Boussinesq, M. J., Ann. Chim. Phys. 29, 349 (1913).

20. Scriven, L. E., Chem. Eng. Science 12, 98 (1960).

21. Edwards, D. A., Brenner, H. \& Wasan, D. T., "Interfacial Transport Processes and Rheology". ButterworthHeinemann, Boston, 1991. 
22. Slattery, J. C., "Interfacial Transport Phenomena". Springer-Verlag, New York, 1990.

23. Stone, H. A., Phys. Fluids A 2, 111 (1990).

24. Wong, H., Rumschitzki, D., and Maldarelli, C., Phys. Fluids 8 (11), 3203 (1996).

25. Avramidis, K.S., and Jiang, T.S., J. Colloid Interface Sci. 147, 262 (1991).

26. Li, D., Slattery, J. C., AIChE Journal 34, 862 (1988).

27. Braun, R. J., Snow, S. A. and Pernisz, U. C., J. Colloid Interface Sci., 219, 225 (1999).

28. Wilson, S. D. R., J. Eng. Math. 16, 209 (1982).

29. Brenan, K. E., Campbell, S. L., and Petzold, L. E., SIAM, Philadelphia (1996)

30. Pawar, Y. P., and Stebe, K. J., Phys. Fluids 8, 1738 (1996).

31. Eggleton, C. D., Pawar, Y. P., and Stebe, K. J., J. Fluid Mech. 385, 79 (1999).

32. Lopez, J. M., and Hirsa, A., J. Colloid and Interface Sci. 206, 231 (1998).

33. Lopez, J. M., and Hirsa, A., Proceedings of the 2nd International Conference on CFD in Minerals and Process Industries, CSIRO, Melbourne, Australia, 1999

34. Stone, H. A., and Leal, L. G., J. Fluid Mech. 230, 161 (1990).

35. Howell, D. J., Hirsa, A. H., Heidari, A. H., and Braun, R. J., to be submitted (2001). 


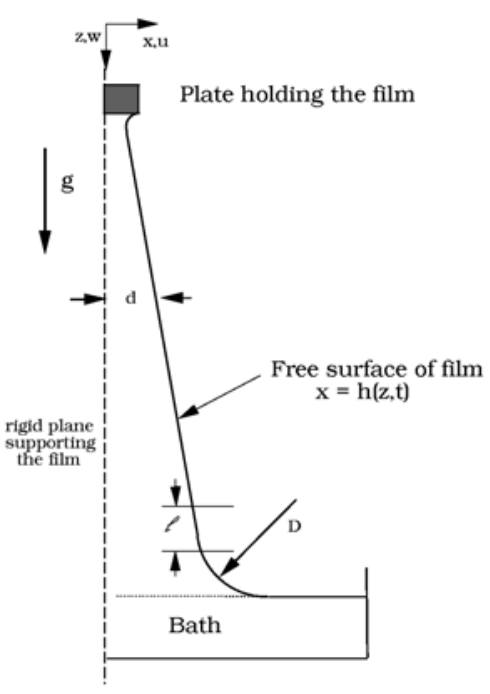

Figure 1. Schematic representation of the model 


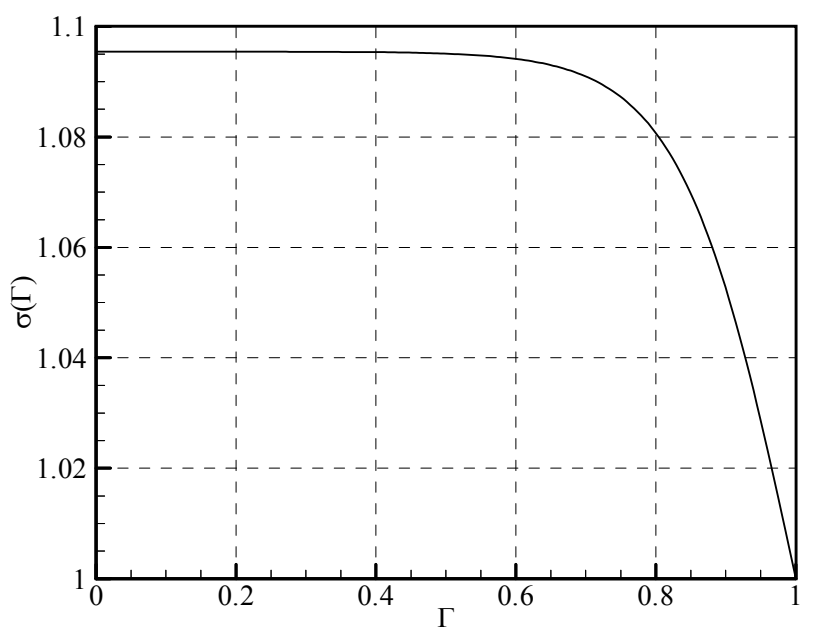

Figure 2. Dimensionless surface tension $\sigma$ as a function of surfactant surface concentration $\Gamma$ based on [9] and [10] with the parameters: $\beta=6.3$ dyne $/ \mathrm{cm}, \alpha=-6.2, \sigma_{m}=66$ dyne $/ \mathrm{cm}$ (18). 

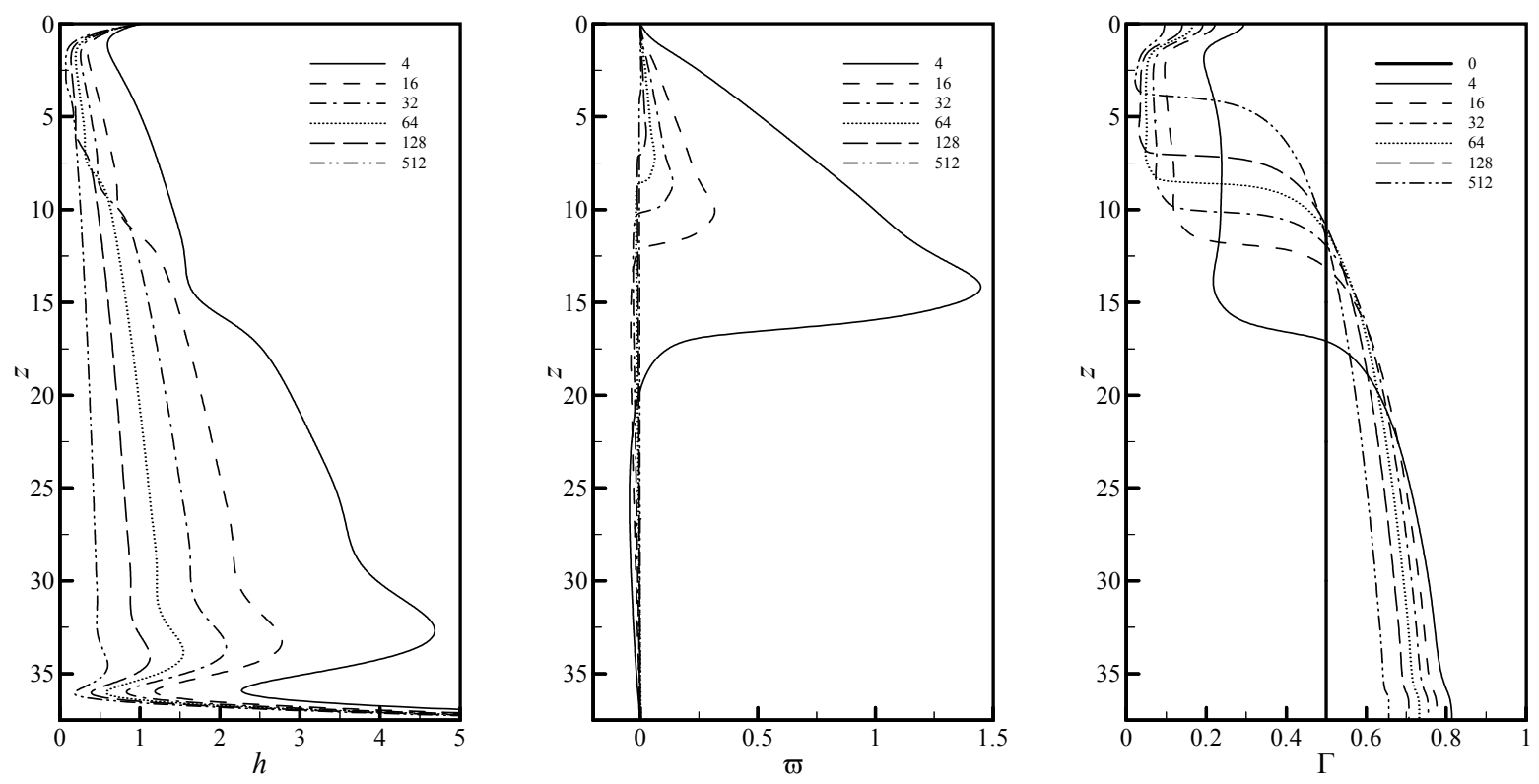

Figure 3. Free surface shape $h$, surface velocity $\varpi$, and surface concentration of surfactant $\Gamma$ for various nondimensional time values for the following parameters

$$
\mathrm{S}=0.16, \mathrm{M}=1500, \mathrm{P}=2700, \hat{\Gamma}=0.50, \gamma_{0}=0.00
$$



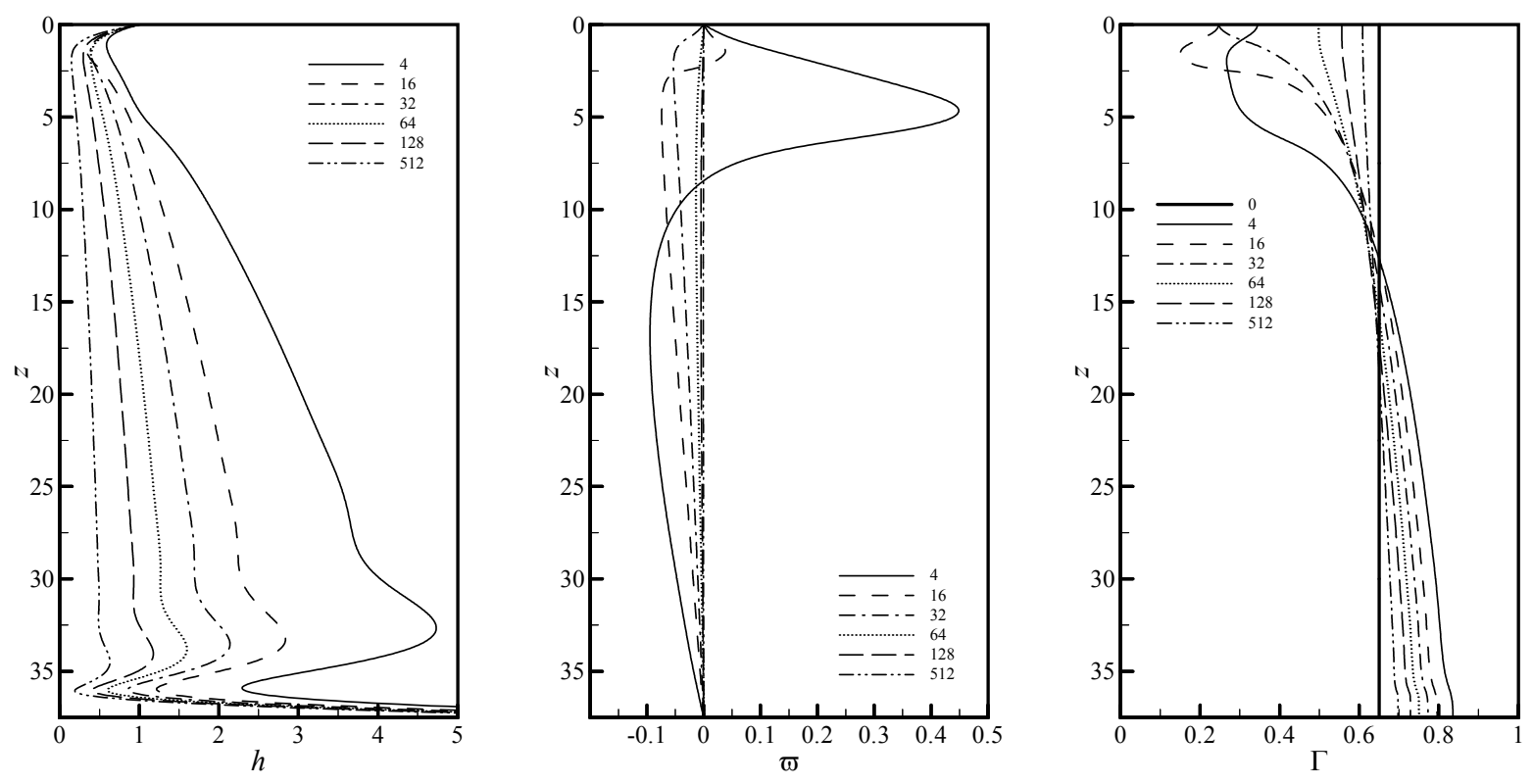

Figure 4. Free surface shape $h$, surface velocity $\varpi$, and surface concentration of surfactant $\Gamma$ for various nondimensional time values for the following parameters

$$
\mathrm{S}=0.16, \mathrm{M}=1500, \mathrm{P}=2700, \hat{\Gamma}=0.65, \gamma_{0}=0 \text {. }
$$



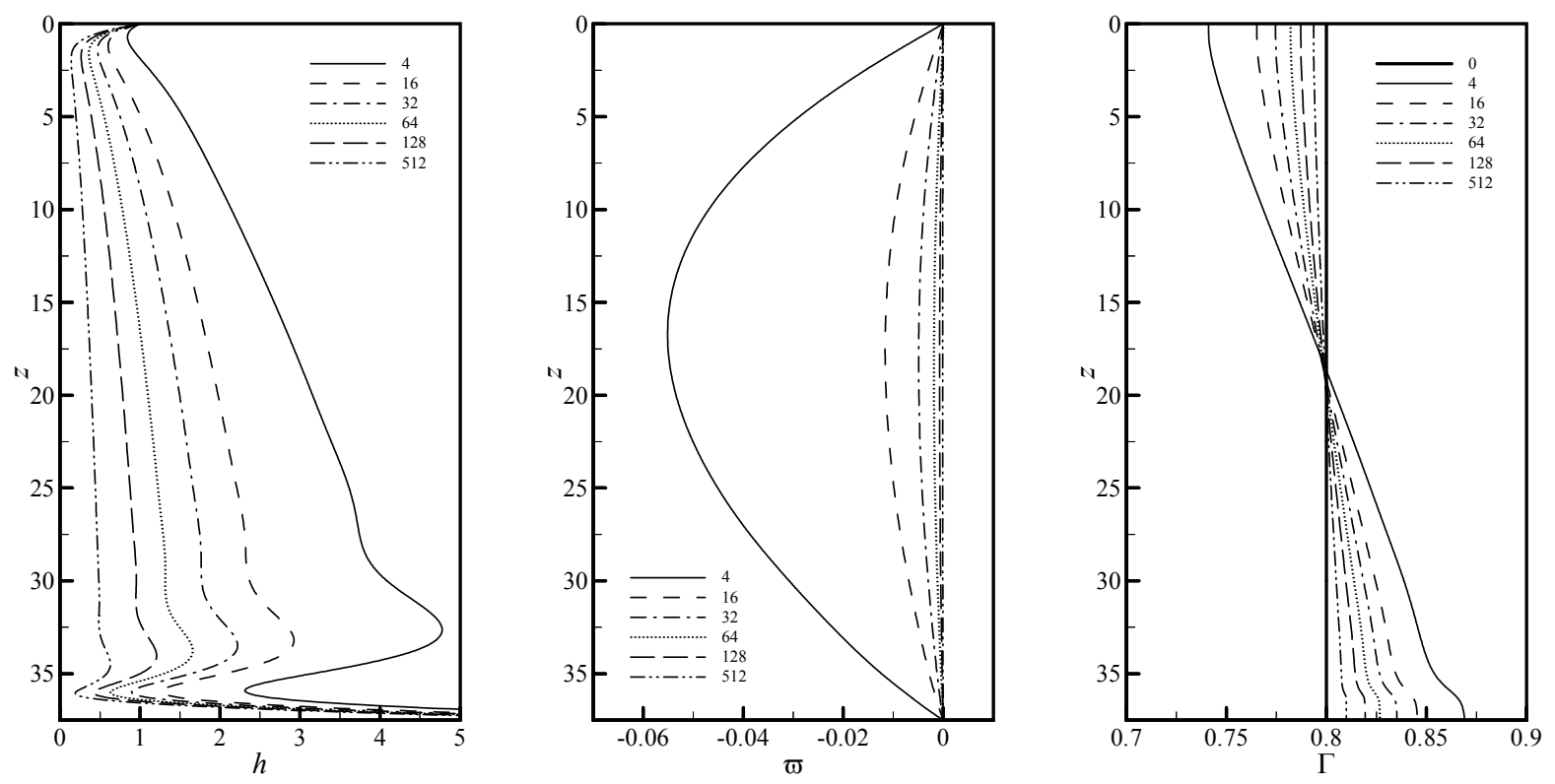

Figure 5. Free surface shape $h$, surface velocity $\omega$, and surface concentration of surfactant $\Gamma$ for various nondimensional time values for the following parameters

$$
\mathrm{S}=0.16, \mathrm{M}=1500, \mathrm{P}=2700, \hat{\Gamma}=0.80, \gamma_{0}=0 .
$$



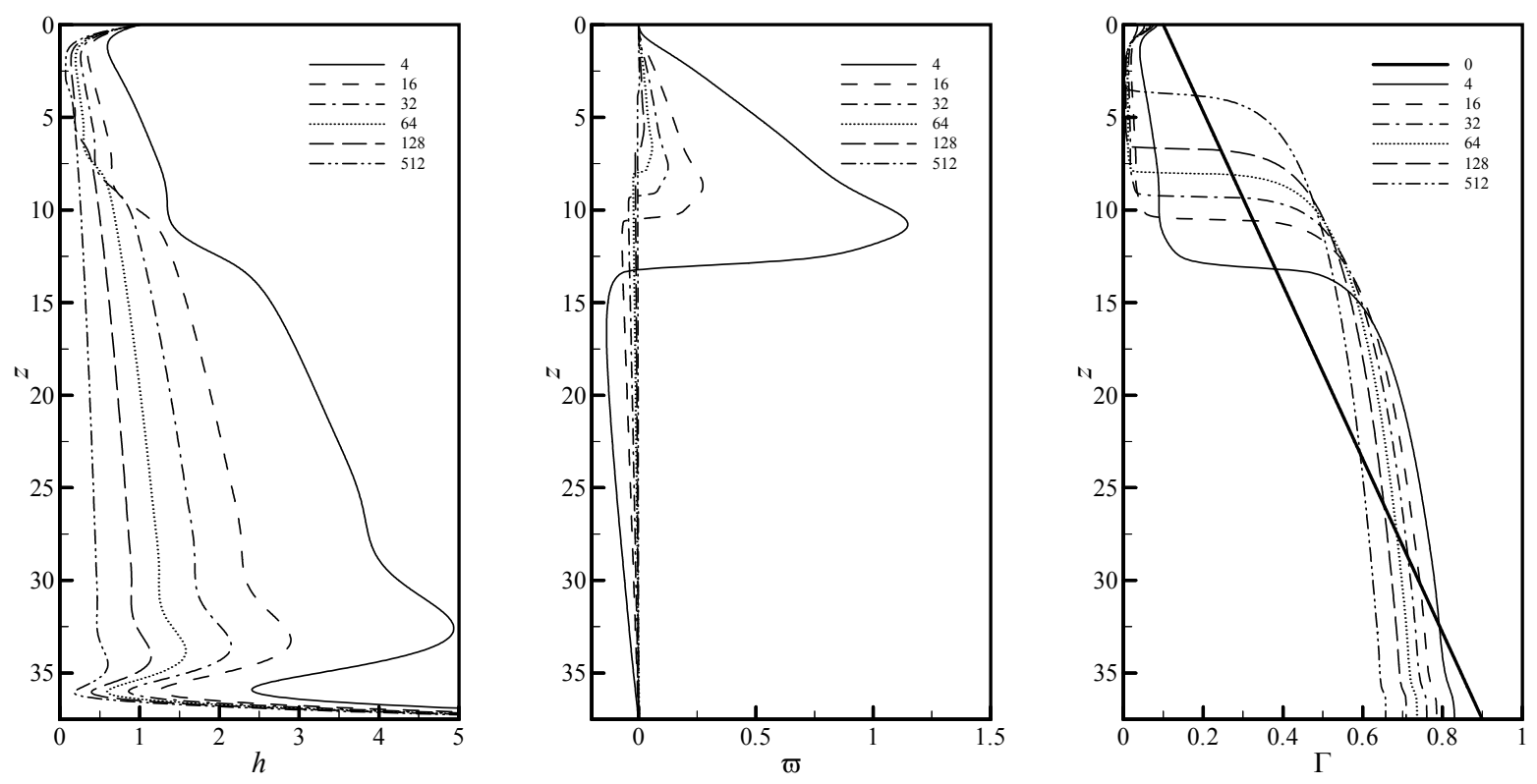

Figure 6. Free surface shape $h$, surface velocity $\varpi$, and surface concentration of surfactant $\Gamma$ for various nondimensional time values for the following parameters $\mathrm{S}=0.16, \mathrm{M}=1500, \mathrm{P}=2700, \hat{\Gamma}=0.50, \gamma_{0}=0.80 / L$ 

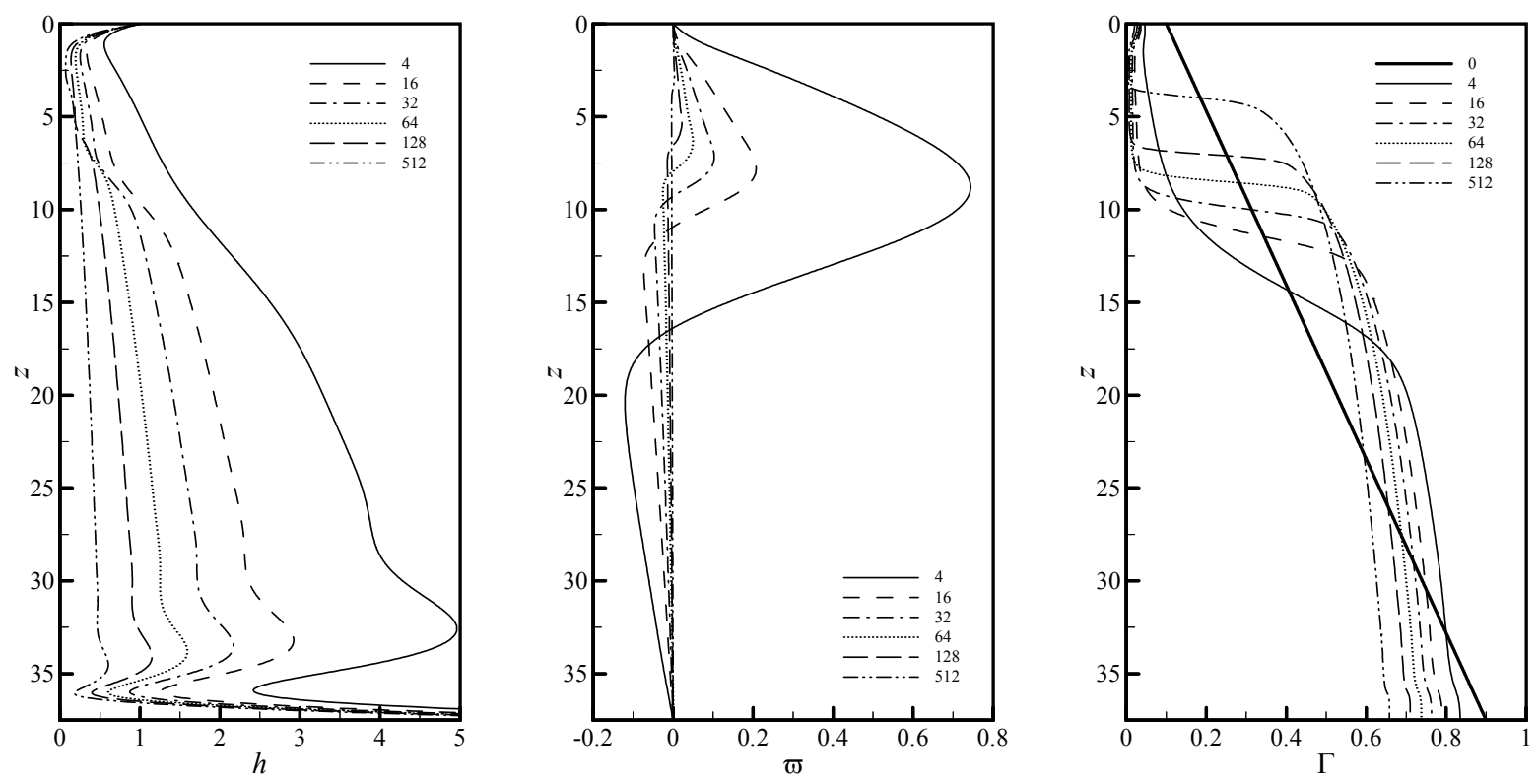

Figure 7. Free surface shape $h$, surface velocity $\varpi$, and surface concentration of surfactant $\Gamma$ for various nondimensional time values for the following parameters $\mathrm{S}=15, \mathrm{M}=1500, \mathrm{P}=2700, \hat{\Gamma}=0.50, \gamma_{0}=0.80 / L$ 

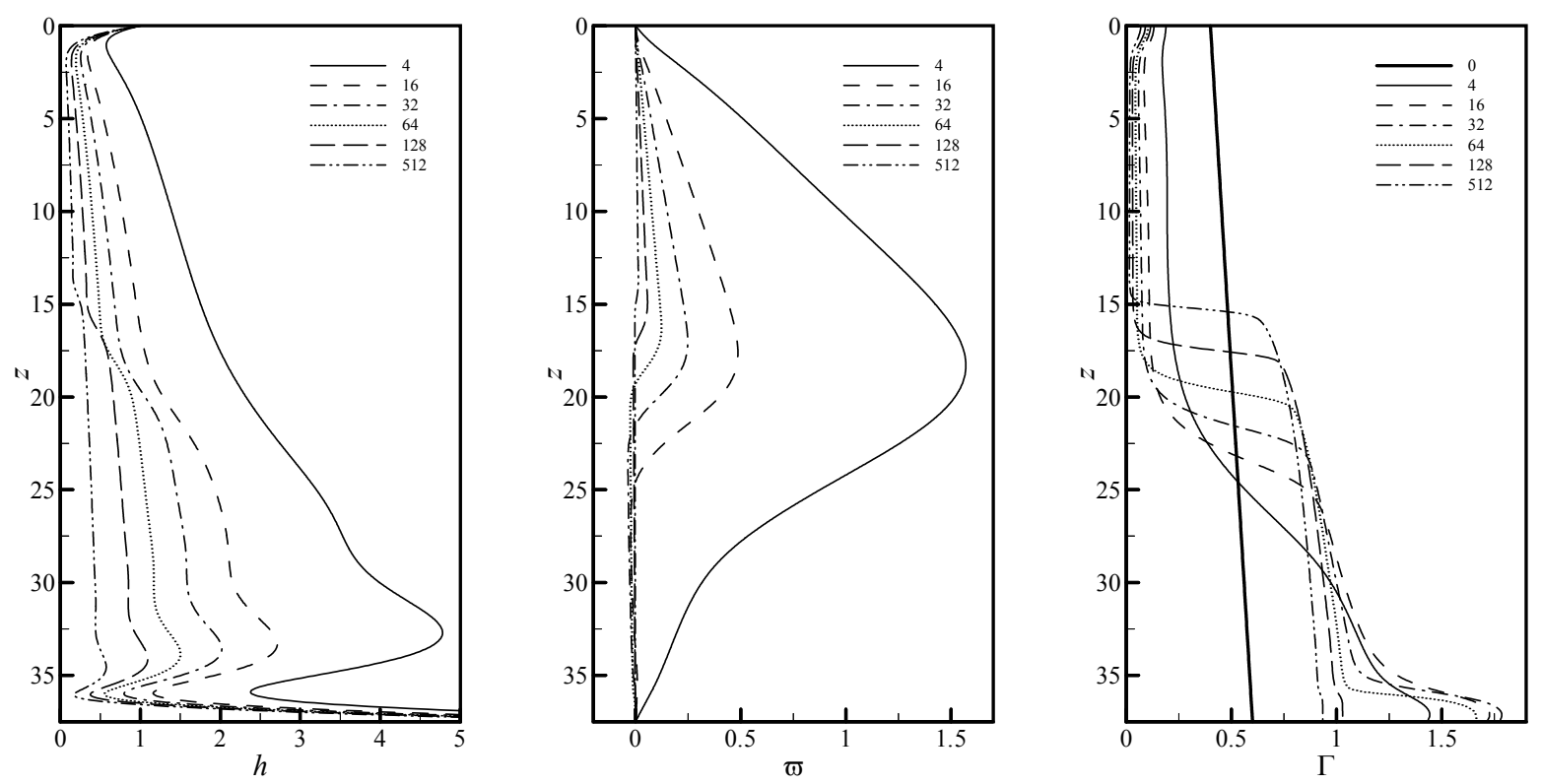

Figure 8. Free surface shape $h$, surface velocity $\varpi$, and surface concentration of surfactant $\Gamma$ for various nondimensional time values for the following parameters

$$
\mathrm{S}=10, \mathrm{M}=50, \mathrm{P}=2700, \hat{\Gamma}=0.50, \gamma_{0}=0.2 / L
$$



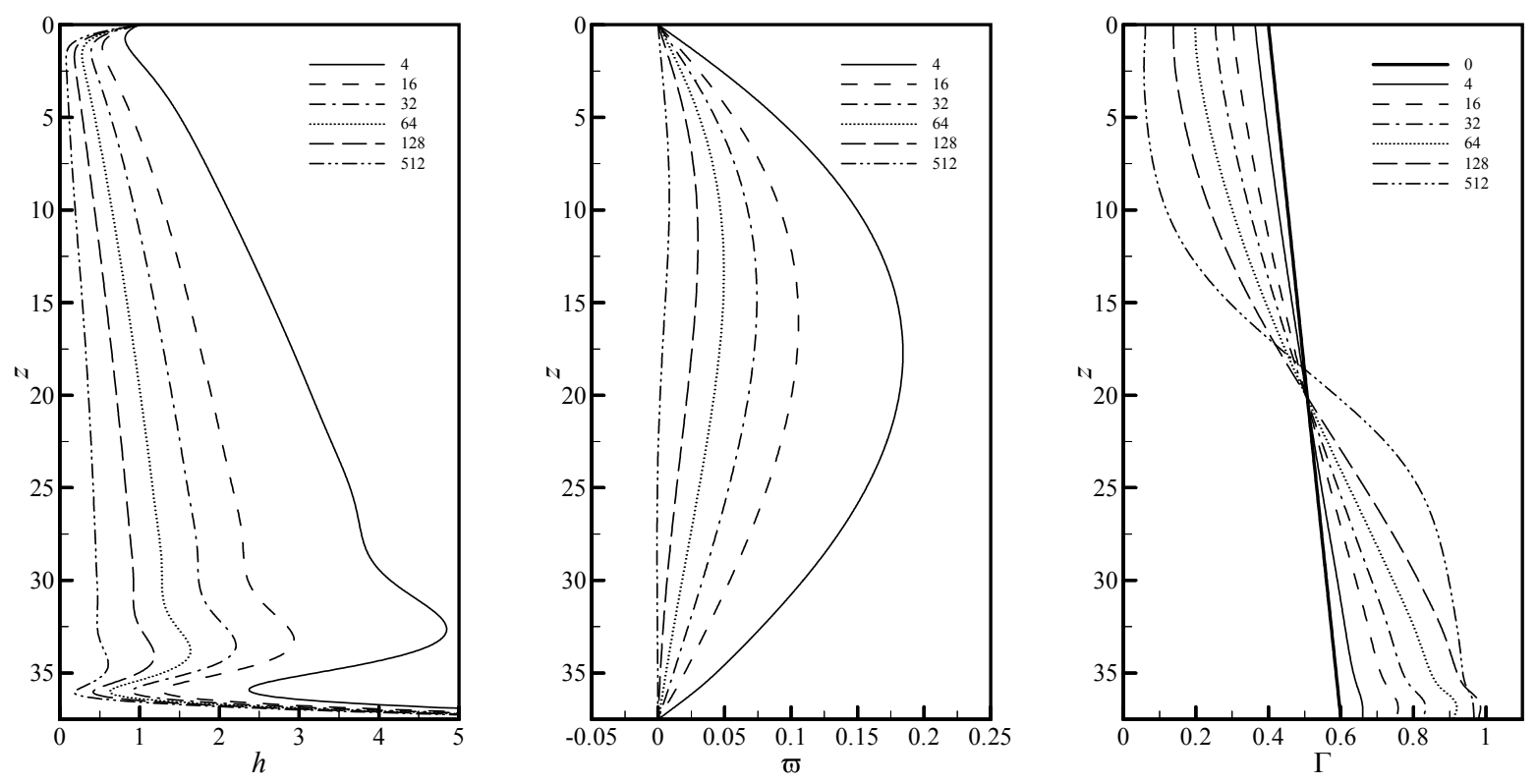

Figure 9. Free surface shape $h$, surface velocity $\varpi$, and surface concentration of surfactant $\Gamma$ for various nondimensional time values for the following parameters

$$
\mathrm{S}=500, \mathrm{M}=50, \mathrm{P}=2700, \hat{\Gamma}=0.50, \gamma_{0}=0.20 / L
$$




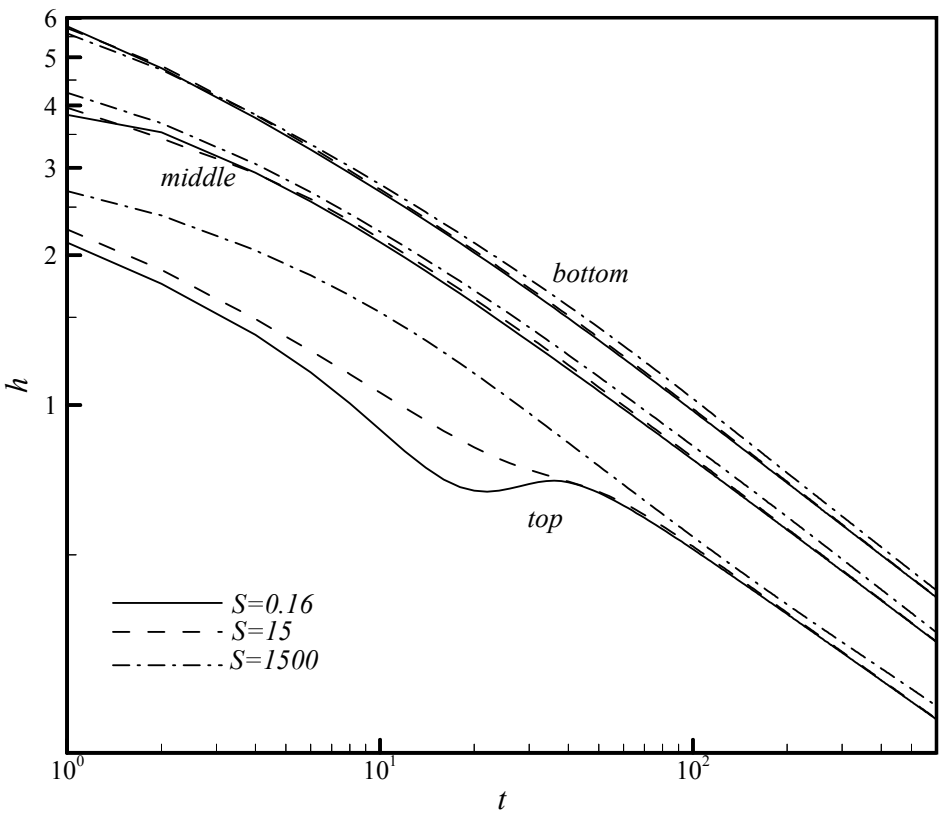

Figure 10. Free surface shape $h$ at the top $(z=L / 4)$, middle $(z=L / 2)$, and bottom $(z=3 L / 4)$ of the film as a function of nondimensional time for various values of modified Boussinesq number and the following parameters: $\mathrm{M}=1500, \mathrm{P}=2700, \hat{\Gamma}=0.50, \gamma_{0}=0.40 / \mathrm{L}$ 